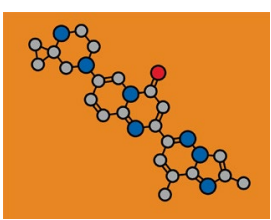

First small-molecule
RNA modulator
approved
p6

Next-generation antibodies to the new coronavirus p9

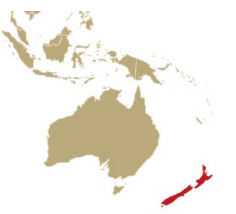

Biotech news from around the world p12

\title{
BTK blockers make headway in multiple sclerosis
}

\author{
By targeting both arms of the immune system, not just B cells, brain-penetrant inhibitors of Bruton's tyrosine kinase \\ could improve on anti-CD20 therapy for patients with multiple sclerosis.
}

S anofi is going all-in on the strategy of targeting Bruton's tyrosine kinase (BTK) to control autoimmunity. In September, the French drug giant completed a $\$ 3.7$ billion takeover of Principia Biopharma, maker of three covalent BTK inhibitors in development for the treatment of various autoimmune conditions.

One particular BTK blocker, the brain-penetrant agent tolebrutinib, is the cornerstone for the acquisition. The two companies forged a previous licensing deal in 2017 over this agent, which entered phase 3 testing last year for both relapsing and progressive forms of multiple sclerosis (MS). Sanofi is now in a three-way contest with Merck KGaA and Genentech, both of which have their own anti-BTK drugs in late-stage trials for MS as well (Table 1).

The idea of targeting BTK in autoimmunity flows from the success of CD20-directed antibodies, such as rituximab (now generic) and Genentech's Ocrevus (ocrelizumab), as therapies for rheumatoid arthritis, MS and other disorders marked by autoreactive B cells. Yet, whereas anti-CD20 drugs completely wipe out all B cells, leaving people prone to opportunistic infections, BTK inhibitors which block a critical enzyme involved in B cell maturation - may more selectively remove unwanted B cells while leaving healthy ones alone.

“That's important. That's what you want," says Peggy Kendall, an allergist and immunologist at Washington University School of Medicine in St. Louis, who has shown in mouse models that autoreactive $\mathrm{B}$ cells depend more on BTK signaling than normal B cells do. Since "the bad B cells are different from the good B cells," she says, "you can target autoreactive B cells without creating B-cell immunodeficiency."

In the context of neuroimmune disorders such as MS, BTK inhibitors, as small molecules, also "have a great opportunity to get into the nervous system where

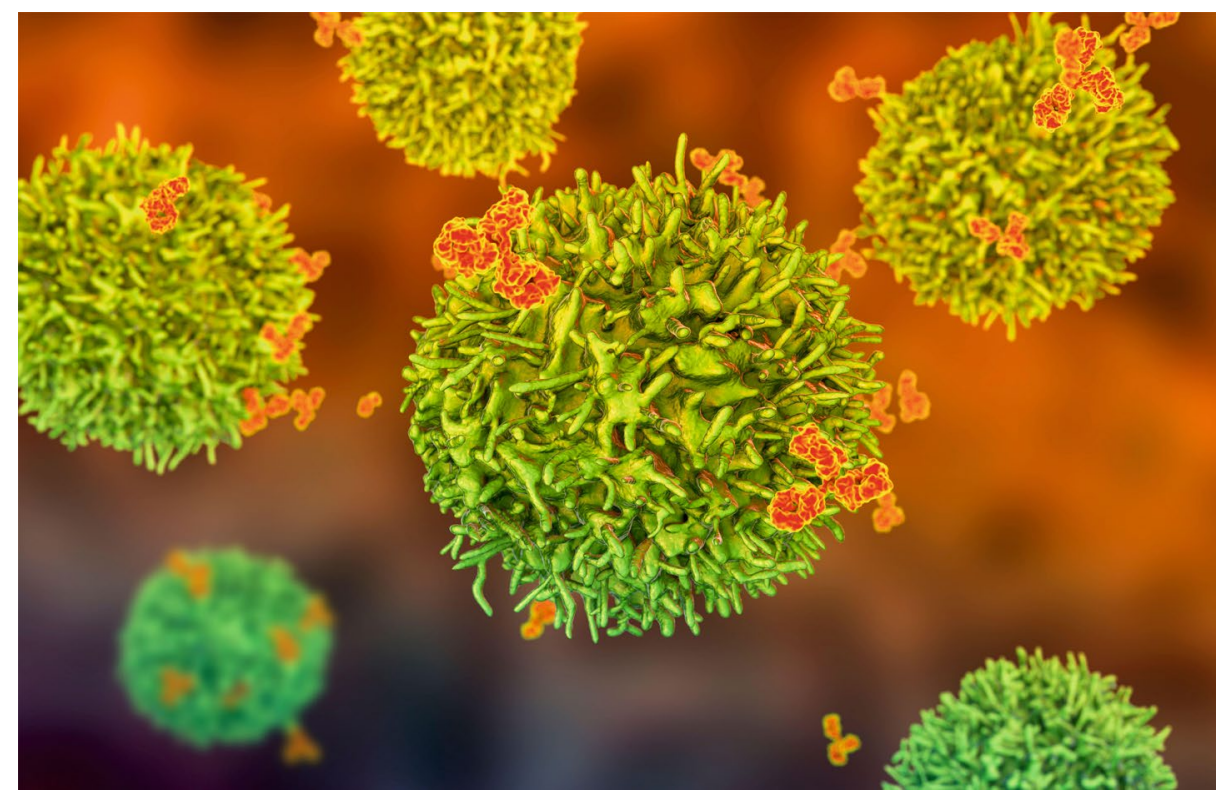

Pathogenic B cells contribute to multiple sclerosis, and BTK inhibitors could stop them maturing. Science Photo Library / Alamy Stock Photo.

antibodies are not that efficient at getting in," notes Stephen Hauser, a neurologist at the University of California, San Francisco (UCSF), who has led trials for CD20- and BTK-directed agents alike. If the small-molecule drugs can reach every brain-resident B cell and "knock out the adaptive inflammation that's overactive in the nervous system," Hauser says, then "I think we can really cure MS."

For BTK inhibitors to work, patients will have to remember to take pills every day a tall ask compared with visiting a doctor's office every six months for an infusion of Ocrevus. But that dosing requirement might be worth it for the added benefit anti-BTK agents could have beyond effects on B cells.

Because BTK signaling is also critical to the activation of various innate immune cells, drugs such as tolebrutinib may serve double duty, modulating both B lymphocytes and proinflammatory macrophages or microglia in one fell swoop. Dietmar Berger, chief medical officer and global head of development at Sanofi, expects those dual functions in the central nervous system (CNS) could ultimately make tolebrutinib the go-to therapy for patients with both relapsing and progressive forms of MS, a disease in which microglia are thought to drive inflammatory lesion formation. "Addressing those mechanisms of the innate immunity might make a big difference," he says.

That's the hope, at least. But microglia can support both inflammatory and reparative functions in the brains of people with MS. And as Luke Healy, a neuroscientist at the Montreal Neurological 
Table 1 | BTK inhibitors in clinical development for autoimmune disorders

\begin{tabular}{|c|c|c|c|c|}
\hline Drug & Lead developer(s) & BTK binding mechanism & Lead indication(s) & Phase \\
\hline Evobrutinib & Merck KGaA & Covalent, irreversible & MS & 3 \\
\hline Tolebrutinib & Sanofi/Principia & Covalent, irreversible & MS & 3 \\
\hline Fenebrutinib & Genentech & Non-covalent, reversible & MS & 3 \\
\hline Rilzabrutinib & Sanofi/Principia & Covalent, reversible & Pemphigus vulgaris, immune thrombocytopenia & 3 \\
\hline Remibrutinib & Novartis & Covalent, irreversible & Urticaria & 2 \\
\hline Tirabrutinib & Gilead Sciences, Ono Pharmaceutical & Covalent, irreversible & Pemphigus vulgaris & 2 \\
\hline Branebrutinib & Bristol Myers Squibb & Covalent, irreversible & Rheumatoid arthritis, lupus, Sjögren's syndrome & 2 \\
\hline Orelabrutinib & InnoCare & Covalent, irreversible & MS & 2 \\
\hline BIIB091 & Biogen & Non-covalent, reversible & MS & 1 \\
\hline AC0058 & Acea Therapeutics & Covalent, irreversible & Lupus & 1 \\
\hline PRN473 & Sanofi/Principia & Covalent, reversible & Dermatology & 1 \\
\hline
\end{tabular}

Institute, points out: "We really still don't have a strong grasp on what role microglia play in the disease, and at what times they play varying roles."

Which function will ultimately be affected by BTK blockade is therefore "a very difficult thing to predict," says Edvard Smith, a molecular geneticist at the Karolinska Institute in Stockholm who isolated the gene encoding BTK in the early 1990s. "It needs to be tested."

BTK inhibitors first rose to prominence in 2013 with the approval of Imbruvica (ibrutinib), a best-selling small molecule drug and now a mainstay of leukemia and lymphoma therapy. Although sold today by AbbVie and Janssen, the drug was originated by scientists at Pharmacyclics (acquired by AbbVie in 2015) and Celera Genomics, who initially evaluated it in mouse models of arthritis before shifting to focus on hematological malignancies.

According to Pharmacyclics founder and former CEO Richard Miller, that decision was largely made for business reasons. With the benefit of hindsight, however, the drug - a covalent inhibitor with activity against BTK and four other TEC-family kinases was probably too toxic for treating chronic, non-life-threatening conditions.

Because of Imbruvica's off-target effects, the drug often causes severe bleeding events and heart rhythm problems, such as atrial fibrillation. Other covalent BTK inhibitors used in cancer - agents such as AstraZeneca’s Calquence (acalabrutinib) and BeiGene's Brukinsa (zanubrutinib) - do show better safety, but their toxicity profiles still make them unsuitable for most non-oncological applications, with the possible exception of treating severe COVID-19 (Box 1).

So when Miller left Pharmacyclics, in 2008, he quickly set about to make a safer alternative - "a cleaner drug," as he puts it. "I thought we could do better." And so Principia was born. The company, cofounded with UCSF chemist Jack Taunton, began making reversible covalent inhibitors of BTK. These drugs retain the specificity advantages of covalently targeting a certain amino acid residue, as Imbruvica does, but without the negative consequences of irreversible off-target activity. "You get high inhibition of BTK without high inhibition of all the other off-targets," explains David Goldstein, CSO at Principia, now a subsidiary of Sanofi.

The first such compound discovered was PRN473. In 2013, Principia began clinically testing an oral version of PRN473 in healthy volunteers. But the drug cleared too quickly from the body. So Goldstein and his colleagues shelved the compound for many years before reformulating it as a topical therapy for immune-mediated skin conditions and jumpstarting clinical development anew in 2020.

In the interim, Principia started advancing another oral reversible covalent BTK blocker, a drug that became known as rilzabrutinib. It was well tolerated, had better pharmacokinetics and showed promise for the treatment of the blistering skin disease pemphigus vulgaris and a blood disorder called immune thrombocytopenia. Phase 3 studies are ongoing in both indications. A phase 2 trial is now also recruiting patients with active IgG4-related disease, a fibro-inflammatory condition that can affect nearly any organ system.

Rounding out the Principia pipeline, the company has a preclinical development program centered around reversible covalent inhibitors of the low-molecular mass protein-7 (LMP7) subunit of the immunoproteasome, a specialized type of protein-degrading machinery that regulates inflammatory responses. However, tolebrutinib, the lead asset, does not bind its target reversibly.

"I can tell you that we tried very hard to make a reversible covalent drug that fully penetrated the CNS compartment - and were unsuccessful doing that with the scaffolds that we had to work with," Goldstein says. So the company moved forward with tolebrutinib, an irreversible covalent agent selected for its ability to cross the blood-brain barrier. In phase 1 testing, clinicians took lumbar punctures from four study volunteers two hours after oral administration of the drug. In the cerebrospinal fluid, they found unbound tolebrutinib in the nanomolar concentration range, a pharmacologically relevant level. Neither Merck KGaA nor Genentech have reported comparable CNS exposure data for their BTK inhibitors.

What Merck KGaA has published suggests that its rival BTK blocker, evobrutinib, functions chemically in much the same way as Imbruvica, engaging irreversibly with the same cysteine residue on the BTK protein. And although evobrutinib looks to be somewhat less potent than Imbruvica, it is far more selective, a property that the company hopes will translate into a better side effect profile.

In phase 2 testing, patients with relapsing MS who received higher doses of evobrutinib showed a trend toward developing fewer brain lesions and experiencing fewer relapses compared with those given a placebo or the Biogen drug Tecfidera (dimethyl fumarate). Evobrutinib treatment was associated with transient elevations in liver enzymes at worst. Tolebrutinib likewise brought about a reduction in MS lesion development in its mid-stage trials, with headaches and cold-like symptoms being the most frequent adverse events. 


\section{Box 1 | BTK inhibitors tapped for COVID-19}

In the early days of the coronavirus pandemic, clinicians were clamoring for therapies that could quell the hyperinflammatory immune response experienced by many people with severe COVID-19. Some reached for corticosteroids, others for cytokine-targeted agents. Louis Staudt and Wyndham Wilson decided to try inhibitors of Bruton's tyrosine kinase (BTK).

The two clinician-scientists from the US National Cancer Institute in Bethesda, Maryland, led a team that administered AstraZeneca’s Calquence (acalabrutinib) off-label to 19 patients hospitalized with severe COVID-19. The therapy seemed to work. It improved oxygenation in most recipients and normalized several measures of inflammation. Studies of blood samples even showed that the drug target, BTK, was highly activated in infected patients compared with healthy controls.

Yet, when AstraZeneca ran two large randomized trials of the drug to test the strategy, adding the BTK blocker to the best available supportive care did not improve clinical outcomes in patients hospitalized with respiratory symptoms of COVID-19.

Wilson chalks the failure up to study design. The trials excluded people who were on mechanical ventilation or required intensive care - that is, the sickest of the

Genentech's fenebrutinib, meanwhile, is an entirely different beast from its covalent competitors. As a non-covalent agent that selectively nestles into a pocket of BTK and dissociates from the target slowly over time, fenebrutinib works to trigger a conformational change in BTK that prevents the addition of chemical adornments needed for enzyme activation. "This binding mode stabilizes an inactive form of BTK and plays a key role in the high selectivity and potency of the compound," says Karin Reif, a former Genentech scientist who helped develop fenebrutinib and now runs a consultancy called KARBio. sick, who stood to gain the most from the therapy - and, in his view, BTK remains "the best target that we have" for quelling a COVID-19-associated cytokine storm.

Not all BTK blockers work the same way, though. And Steven Treon, an oncologist at the Dana-Farber Cancer Institute in Boston, suspects that drugs targeting both BTK and another enzyme called hematopoietic cell kinase (HCK) could have an enhanced effect.

Treon previously found that the AbbVie/Janssen BTK inhibitor Imbruvica - which, unlike Calquence, but like BeiGene's anti-BTK agent Brukinsa (zanubrutinib), also hits HCK - protects against lung injury and improves COVID19-related symptoms among patients receiving the drug for Waldenström macroglobulinemia, a rare lymphoma. He is now involved in controlled trials of both Imbruvica and Brukinsa for COVID-19, and he says he remains "cautiously optimistic" that those other drugs may succeed where Calquence failed.

"We have kind of lumped these all into one class," Treon says, "but the individual spectrum of their kinase inhibition plays a very important role." And while selectivity for BTK may be a hindrance in the management of COVID-19, it is proving to be a must-have for drugmakers when it comes to treating autoimmune conditions, such as MS.

Genentech initially evaluated the drug for the treatment of hives, rheumatoid arthritis and lupus before deciding to jump right into large phase 3 trials for patients with MS last year. Although those trials won't begin to yield results until 2024 - about a year after studies involving evobrutinib and tolebrutinib read out - Genentech's head of neuro-immunology, Kathleen Hawker, suspects that fenebrutinib might have an edge over the covalent BTK inhibitors in selectivity and thus safety.

As the company behind the anti-CD20 antibody Ocrevus, Genentech is also well positioned to offer combination regimens of B-cell-directed MS agents. Mouse experiments from neurologist Martin Weber's group at the University Medical Center Göttingen in Germany suggest that BTK inhibitors might be better at preventing the development of pathogenic $B$ cells than at controlling B cells with established disease-driving properties.

Therefore, Weber, who serves on the steering committee for the fenebrutinib trials and has led testing of evobrutinib, thinks that drugs like fenebrutinib might work best if teed up with a dose of B-cell-eliminating Ocrevus to clear the deck. "In my mind, that's the true positioning for those drugs," he says.

According to Hawker, Weber's proposal is "something that we're interested in." But first, she says, "we have to have some data from fenebrutinib [as a monotherapy] before we can talk about combination or sequential" dosing schemes.

More immediately, Hawker and others hope to resolve the extent to which microglial targeting underpins the therapeutic benefits of BTK inhibition. Some clues are emerging from experimental model systems.

In mice, neuroscientist Ethan Hughes and his colleagues at the University of Colorado School of Medicine in Aurora have shown that treatment with a tolebrutinib-like compound can stop microglia from rapidly engulfing myelin sheaths and starting the process of demyelination after the delivery of disease-causing antibodies onto the cortical surface. And in transgenic tadpoles, Bernard Zalc, a neuroscientist at the Brain and Spine Institute in Paris, reported that an evobrutinib-like drug can even promote myelin repair.

"It's the first demonstration that these inhibitors can work on innate immunity and favor remyelination," Zalc says. Now, with new brain imaging modalities and better biomarkers of microglial activity, researchers hope to confirm whether the same is true in people as well.

Elie Dolgin

Somerville, MA, USA

Published online: 11 January 2021

https://doi.org/10.1038/s41587-020-00790-7 